\title{
Aspects of the ladder approximation bound state spinor-spinor Bethe-Salpeter equation
}

\section{B.J. Brennan}

Following a summary of relevant features of the bound state spinorspinor Bethe-Salpeter equation, the normalisation of Bethe-Salpeter amplitudes is examined, and it is shown that for at least one ladder approximation spinor-spinor solution, the two commonly written forms of the normalisation condition appear to be inequivalent.

We then examine symmetries of the coupling parameter $\lambda$, and demonstrate that $\lambda$ is an even function of both the bound state mass and the difference between the masses of the constituent particles.

Next we examine the unequal-mass spinor-spinor Bethe-Salpeter equation for systems with zero bound state mass. Coupled radial equations are derived, and their symmetries examined. The numerical behaviour of $\lambda$ as a function of the ratio of the constituent particle masses is examined for known analytical equal-mass solutions.

A perturbation method is then employed to investigate the behaviour of $\lambda$ for small values of the exchange boson mass.

After this certain aspects of the equal-mass Bethe-Salpeter equation are examined. For systems with zero bound state mass, we consider the analysis of a search for $S V$ sector solutions, and also examine solutions for a model involving the exchange of both vector and pseudoscalar bosons. Finally we consider the solution of the radial equations for non-zero

Received 11 July 1975. Thesis submitted to the University of Auckland, February 1975. Degree approved, July 1975. Supervisor: Associate Professor R.F. Keam. 
bound state mass, using numerical methods together with a perturbation approach. 